\title{
Ausbildung als Schlüssel für eine adäquate Behandlung von Elektroaltgeräten in Lateinamerika
}

\author{
Stefan Salhofer - Elisabeth Schmied - Aleksander Jandric
}

Angenommen: 21. Juni 2021 / Online publiziert: 12. Juli 2021

(C) Der/die Autor(en) 2021

\begin{abstract}
Zusammenfassung Wie in anderen Teilen der Welt wächst auch in Lateinamerika die Ausstattung der Haushalte mit elektrischen und elektronischen Geräten. Die daraus resultierenden Elektroaltgeräte treffen auf wenig entwickelte abfallwirtschaftliche Strukturen. In den meisten Ländern Lateinamerikas ist die getrennte Sammlung von Abfällen kaum entwickelt, Anlagen für eine spezifische Behandlung von Abfällen wie Elektroaltgeräte fehlen weitgehend. Sammlung und Behandlung werden

Zurzeit bestehen in beiden Ländern kaum Möglichkeiten für eine fachliche Ausbildung in diesem Bereich. Um den zukünftig zu erwartenden Bedarf abzudecken wurde das Projekt LaWEEEda (im Erasmus+Programm gefördert) ins Leben gerufen. An den vier beteiligten Universitäten wurde ein akademisches Programm für Abfallwirtschaft und Behandlung von Elektroaltgeräten sowie ein berufsbegleitendes Ausbildungsprogramm für PraktikerInnen in diesem Bereich etabliert.
\end{abstract} hauptsächlich durch den informellen Sektor durchgeführt.

In diesem Beitrag werden beispielhaft zwei Länder näher betrachtet.

Nicaragua, als eines der am wenigsten entwickelten Länder, und Brasilien, ein großes und teilweise industrialisiertes Land, das allerdings keine entwickelten Strukturen zur Sammlung und Behandlung von Abfällen aufweist. In Nicaragua gibt es derzeit keine spezifischen gesetzlichen Regelungen für den Umgang mit Elektroaltgeräten und nur einzelne, kleinere Initiativen zur Sammlung sowie einen Behandlungsbetrieb in Managua.

In Brasilien dominiert der informelle Sektor die Sammlung und Behandlung von Elektroaltgeräten. Gesetzliche Regelungen werden seit vielen Jahren diskutiert. Eine Branchenregelung soll Herstellerverantwortung einführen, allerdings sind die Rollen und Verpflichtungen der Stakeholder nicht geklärt. Im bestehenden Entsorgungssystem spielen Kooperativen eine wichtige Rolle.

Für die Planung, Umsetzung und das Monitoring abfallwirtschaftlicher Systeme ist ExpertInnenwissen unerlässlich.

\footnotetext{
ao. Univ.-Prof. DI Dr. S. Salhofer $(\bowtie)$. DI E. Schmied · DI A. Jandric Department Wasser-Atmosphäre-Umwelt, Institut für Abfallwirtschaft, Universität für Bodenkultur Wien, Muthgasse 107, 1190 Wien, Österreich stefan.salhofer@boku.ac.at
}

Schlüsselwörter Elektroaltgeräte . Recycling · Akademische Ausbildung • Berufsbegleitende Ausbildung . Nicaragua $\cdot$ Brasilien

\section{Education as a key for the} appropriate treatment of waste electrical and electronic equipment

Abstract As in other parts of the world, households in Latin America are increasingly equipped with electrical and electronic appliances. The resulting waste electrical and electronic equipment (WEEE) encounters poorly developed waste management structures. In most Latin American countries, the separate collection of waste is hardly developed, and facilities for specific treatment of waste such as WEEE are largely lacking. Collection and treatment are mainly carried out by the informal sector.

This paper takes a closer look at two countries as examples, Nicaragua, as one of the least developed countries, and Brazil, a large and partly industrialised country, which, however, does not have developed waste collection and treatment structures. In Nicaragua, there are currently no specific legal regulations for the handling of WEEE and only individual, smaller initiatives for collection, as well as a treatment facility in Managua.

In Brazil, the informal sector dominates the collection and treatment of
WEEE. Legal regulations have been discussed for many years. An industry regulation is supposed to introduce producer responsibility, but the roles and obligations of the stakeholders are not clear. In the existing waste management system, cooperatives play an important role.

Expert knowledge is essential for planning, implementing and monitoring waste management systems. Currently, there are hardly any opportunities for specialised training in this field in both countries. In order to meet the expected future needs, the LaWEEEda project (funded by the Erasmus+programme) was launched to establish an academic programme for waste management and treatment of WEEE as well as an in-service training programme for practitioners in this field at the four participating universities.

Keywords Waste electrical and electronic equipment · Recycling . Academic education - Continuing professional education - Nicaragua . Brazil

\section{Einleitung}

Der geordnete Umgang mit Elektroaltgeräten (EAGs) ist in Lateinamerika zu einem bedeutsamen Thema geworden. Wie in anderen Teilen der Erde ist auch hier $\mathrm{zu}$ beobachten, dass Haushalte und Betriebe zunehmend mit elektrischen und elektronischen Geräten ausgestattet sind. Dies führt zu einer Zunahme der zu entsorgenden Geräte. In den meisten Ländern Lateinamerikas ist dieser Abfallstrom noch nicht rechtlich geregelt, von den 31 Ländern Mittel- und Südamerikas haben nach Forti et al. (2020) derzeit 7 Länder Regelungen. Mexico, Costa Rica, Kolumbien und Peru gelten als Vorreiter, weitere Länder mit Fortschritten bei der Umsetzung sind Brasilien, Chile und Argentinien. 
Davon abgesehen fehlt es in den Ländern Lateinamerikas an abfallwirtschaftlicher Infrastruktur, der Informelle Recyclingsektor dominiert die Sammlung und teilweise auch die Behandlung von EAGs. Dies führt zu Praktiken, die als „Rosinenpicken“ bezeichnet werden, bei denen nur die wertvollen Teile gewonnen werden und der Rest ungeordnet entsorgt wird. Diese Praktiken sind umweltverschmutzend, stellen aber auch eine gesundheitliche Gefahr für ArbeiterInnen und AnrainerInnen dar. Der Anteil der Elektroaltgeräte, der einer geordneten Sammlung und Verwertung zugeführt wird, wird von Forti et al. (2020) mit $1 \%$ für die Karibik, $3 \%$ für Mittelamerika und $0,7 \%$ für Lateinamerika angegeben.

In Lateinamerika konnten in den letzten 5 bis 10 Jahren Fortschritte bei der Entwicklung von Rechtsvorschriften beobachtet werden. Diese Fortschritte beschränken sich jedoch auf wenige Länder, da die Entwicklung in Lateinamerika Zeit braucht und nur wenige Länder es geschafft haben, EAG-spezifische Rechtsvorschriften zu erlassen. Abgesehen von Mexiko, Costa Rica, Kolumbien und Peru - den wahrscheinlich führenden Kräften in der Region für umweltverträgliches EAG-Management, die im Jahr 2020 an der Verbesserung der bereits etablierten Systeme arbeiten - schufen nur Brasilien und Chile die Grundlagen, um mit der Umsetzung eines formalen Regelwerks für EAGs zu beginnen.

Brasilien hat Ende 2019 das „Sektorale Abkommen für die Umsetzung des Reverse-Logistik-Systems für Elektroaltgeräte aus Haushalten“ zur öffentlichen Konsultation veröffentlicht, dessen formale Unterzeichnung für 2020 erwartet wurde. Nach der Verabschiedung des „Rahmengesetzes über die Abfallwirtschaft, die erweiterte Herstellerverantwortung und die Förderung des Recyclings“" im Jahr 2016 arbeitet Chile nun an der spezifischen EAGVerordnung, die Sammlungs- und Recyclingziele enthalten und die Richtlinien für die Implementierung von formalen Sammelsystemen festlegen wird.

Sieben Jahre nach der Umsetzung des Dekrets 1512 für Computer, Drucker und andere Office-Geräte-Abfälle (MAVDT 2010) arbeitet Kolumbien an einer neuen Verordnung, um die erweiterte Herstellerverantwortung auf alle EAG-Kategorien auszudehnen und Anpassungen am integrierten Managementsystem für EAGs vorzunehmen, wobei die Erfahrungen und Richtlinien des WEEE-Gesetzes 1672 und der nationalen Politik für das WEEE-Management berücksichtigt werden (Forti et al. 2020).

In Peru ist es bereits fünf Jahre her, dass das erste Regulierungssystem für die Entsorgung von EAGs eingeführt wurde, derzeit werden die bisherigen Erfahrungen ausgewertet, um Regelungslücken zu schließen und Anpassungen an die allgemeine Abfallwirtschaftsstrategie des Landes vorzunehmen. Es wird erwartet, dass die überarbeitete Verordnung in Kürze veröffentlicht wird. Sie wird auch den Umfang der EAG-Kategorien mit einem verpflichtenden Sammelziel für kleine und große Haushaltsgeräte und insbesondere Kühlgeräte erweitern. Mexiko plante für 2020 eine Überprüfung der aktuellen Verordnung nach ihrer ersten fünfjährigen Laufzeit und hat mehrere Studien durchgeführt, um die Zuständigkeiten der beteiligten Akteure neu zu definieren, definierte Gerätekategorien festzulegen und verbindliche Sammelziele zu setzen und so die gesammelten und formal recycelten Mengen zu erhöhen. Costa Rica hat seine anfänglichen Herausforderungen, die durch widersprüchliche Regelungen entstanden sind, endlich überwunden und konzentriert sich nun auf die Verbesserung der Umsetzung der aktuellen Regelung.

Nach zahlreichen erfolglosen Initiativen und Gesetzesvorhaben für Elektroaltgeräte auf Bundes- und Provinzebene hat Argentinien seinen Ansatz geändert und ein Gesetz über die erweiterte Herstellerverantwortung für mehrere Abfallkategorien entworfen. Das Gesetz wird derzeit im Kongress diskutiert.

\section{Situation in Nicaragua}

\subsection{Regelungen in der Abfallwirtschaft}

Die wichtigste gesetzliche Regelung auf nationaler Ebene in Nicaragua, die die Behandlung und Entsorgung von Siedlungsabfällen regelt, ist das Exekutivdekret Nr. 47-2005 „Nationale Politik zum integrierten Abfallmanagement" (SGIRS 2005). Das Exekutivdekret enthält allgemeine Ziele der nationalen Politik in Bezug auf die Abfallwirtschaft sowie eine Liste von 17 spezifischen Zielen, z.B. Schutz der öffentlichen Gesundheit der BürgerInnen, die direkt und indirekt in die Abfallwirtschaft involviert sind, und Vermeidung und
Verringerung der abfallbedingten Verschmutzung von Boden, Wasser und Luft. Allerdings sind in der Durchführungsverordnung weder verpflichtende Anforderungen an die Abfallbehandlung und -entsorgung noch andere Arten von spezifischen Vorschriften enthalten.

Das Management von Elektroaltgeräten ist mit dem „Sondergesetz für die integrale Bewirtschaftung von gefährlichen und nicht gefährlichen Abfällen“ geregelt (ANdD 2014). Die EAGs werden ausschließlich im Artikel 64 des Sondergesetzes erwähnt, in dem nur das zuständige Ministerium und die allgemeine Zielsetzung zur Verringerung, Recycling, und Beseitigung von EAGs erwähnt werden. Neben den nationalen Gesetzen ist Nicaragua Unterzeichner der Basler Konvention, die Vorgaben der Basler Konvention sind jedoch nicht in nationalen Gesetzen umgesetzt worden. Auf Initiative internationaler Organisationen gibt es immer wieder Versuche, ein System der erweiterten Herstellerverantwortung (eng. Extended Producer Responsibility) für EAGs zu erstellen. Allerdings waren diese Versuche bisher nicht erfolgreich.

\subsection{Abfallwirtschaft im Überblick}

Nicaragua ist ein Land in Mittelamerika zwischen Pazifik und Karibischem Meer mit einer Fläche von ca. $130.000 \mathrm{~km}^{2}$ (etwas größer als Österreich und die Schweiz zusammen) und 6,5 Mio. EinwohnerInnen. Nach einem Bericht der Inter-American Development Bank (IADB 2011) lag das Aufkommen an festen Siedlungsabfällen bei $0,73 \mathrm{~kg} / \mathrm{EW} . \mathrm{a}$, was ca. 1,5 Miot/a entspricht. Der Anschlussgrad der kommunalen Abfallsammlung ist in Nicaragua im Vergleich zu Ländern mit ähnlichem BIP in Afrika und Asien relativ hoch. Er beträgt ca. $92 \%$, wobei er in den beiden größten Städten Managua und Leon nahe bei $100 \%$ liegt, und in den ländlichen Gebieten niedriger ist (IADB 2011).

In Nicaragua gibt es drei Arten von Abfallsammelsystemen, die oft parallel für verschiedene Abfallarten arbeiten. Rund $74 \%$ der Bevölkerung werden durch öffentliche kommunale Abfallsammelsysteme entsorgt, weitere $22 \%$ der Bevölkerung werden von privaten Sammelunternehmen abgedeckt und die restlichen $4 \%$ von Kooperativen (IADB 2011). In Bezug auf die Zusammensetzung ist der kommuna- 


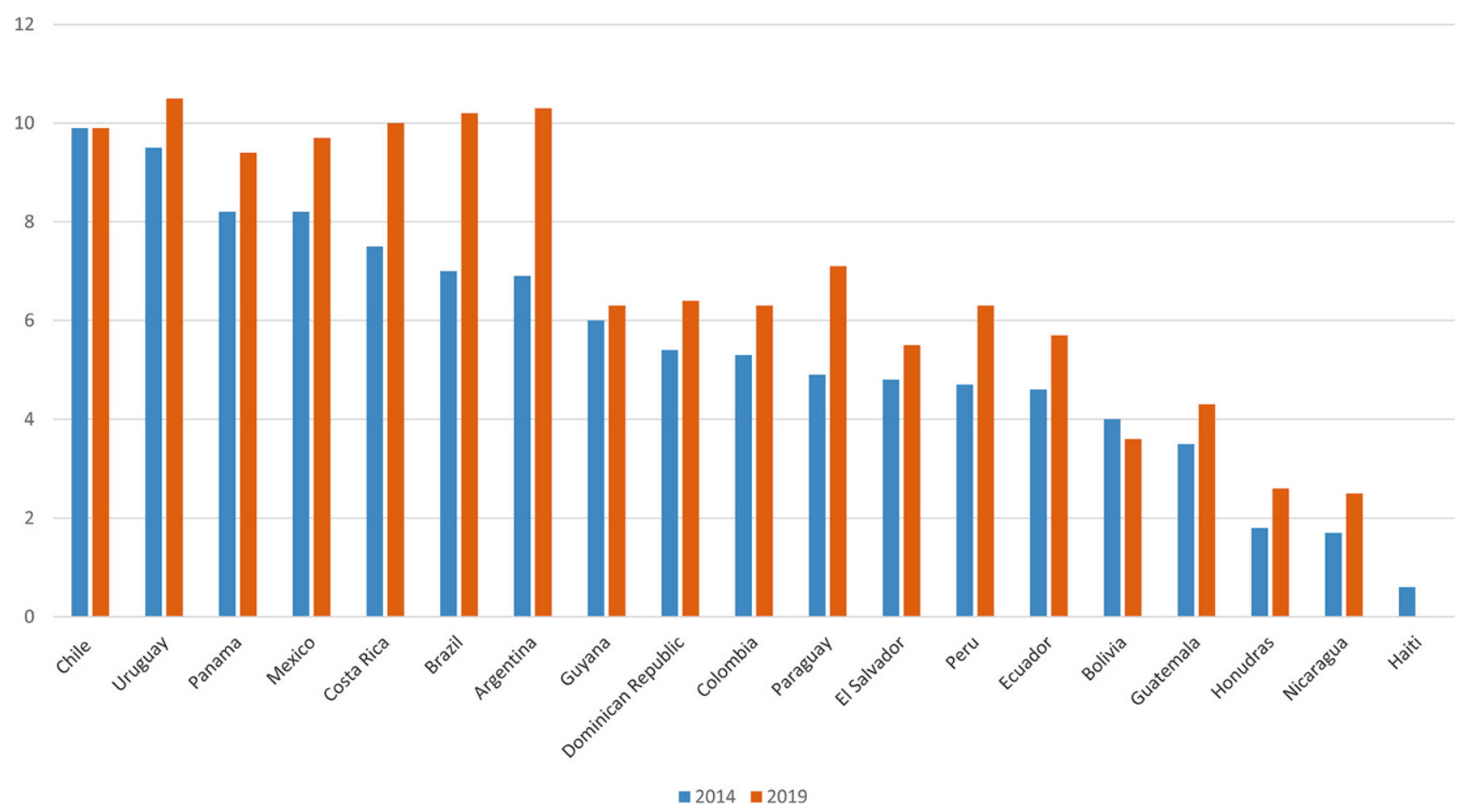

Abb. 1 EAG-Aufkommen in Lateinamerika kg pro Person (Baldé et al. 2015; Forti et al. 2020)

le Siedlungsabfall in Mittelamerika im Allgemeinen durch hohe Feuchtigkeit und einen niedrigen Heizwert gekennzeichnet. Die durchschnittliche Zusammensetzung der festen Siedlungsabfälle in Lateinamerika und der Karibik setzt sich aus ca. $52 \%$ organischen Abfällen, $13 \%$ Papier und Pappe, $12 \%$ Kunststoffen, $4 \%$ Glas, 3\% Metall und $15 \%$ Sonstiges zusammen (Kaza et al. 2018). Die Behandlung und Entsorgung der gesammelten festen Siedlungsabfälle ist wahrscheinlich das schwächste Glied in der nicaraguanischen Abfallwirtschaft. Mehr als $60 \%$ der anfallenden festen Siedlungsabfälle landen auf unkontrollierten Deponien, weitere $20 \%$ auf kontrollierten Deponien, 8\% werden in den Gärten der Privathaushalte verbrannt, und der Verbleib der restlichen $12 \%$ ist unbekannt (Arce et al. 2010).

\subsection{Umgang mit Elektroaltgeräten}

Im Jahr 2019 sind in Nicaragua rund $16.000 \mathrm{t}$ oder $2,5 \mathrm{~kg} /$ EW.a an EAGs angefallen. Obwohl es mit Honduras und Haiti am Ende der Liste der lateinamerikanischen Länder in Bezug auf das Aufkommen von EAGs steht, zeigt Nicaragua einen Anstieg im Aufkommen von EAG von $9 \%$ pro Jahr (Baldé et al. 2015; Forti et al. 2020). Diese vergleichsweise kleinen Mengen, gekoppelt mit rasantem Zuwachs, werden bei unsachge- mäßer Behandlung zu ernsthaften Umweltbelastungen führen (Abb. 1).

Da es keine Infrastruktur für die getrennte Sammlung von Abfällen gibt, erfolgt die Sammlung von EAGs auf alternativen Wegen. Hochwertige Elektronik-Altgeräte, z.B. Mobiltelefone, Smartphones, Tablets und PCs, werden von informellen SammlerInnen gesammelt, manchmal sogar gegen Vergütung. Weniger wertvolle und sperrige EAGs werden entweder ebenfalls von informellen SammlerInnen erfasst, gemeinsam mit anderen Siedlungsabfällen gesammelt oder auf unkontrollierten Deponien entsorgt. In den beiden letzten Szenarien landen die EAGs auf unkontrollierten Deponien, wo informelle Recycler Wertstoffe, in der Regel Metalle, manchmal auch Kunststoffe, aussortieren und vermarkten.

\subsection{Lokale Initiativen}

In der Hauptstadt Managua sollen zumindest 3000 Familien mit dem Sammeln und Recyceln von Abfällen ihren Lebensunterhalt verdienen (laWEEEda 2017), viele davon nach wie vor auf der Deponie „La Chureca“. Diese Deponie besteht seit 1971 , dort werden $90 \%$ des Abfalls von Managua ohne Vorbehandlung abgelagert. Die La-Chureca-Deponie ist mittlerweile zu einer der 50 größten Mülldeponien der Welt geworden
(Kaza et al. 2018). Im Jahr 2009 verdienten dort 1500 bis 2000 Menschen, die als "Churequeros“ bezeichnet werden, ihren Lebensunterhalt. Im Jahr 2009 wurde die Deponie im Rahmen eines groß angelegten Entwicklungsprojekts der AECID (Spanische Agentur für Internationale Zusammenarbeit für Entwicklung) und der Stadtverwaltung saniert und mit einer Recyclinganlage ausgestattet. Im Zuge dessen wurden u. a. Häuser für 258 Familien gebaut, die bisher auf dem Gelände gelebt haben. Einige der Churequeros begannen, sich zu organisieren und gründeten eine $\mathrm{Ge}-$ werkschaft und Kooperativen (z. B. Cooperativa La Chureca Guardabarranco und Cooperativa de Mujeres Recicladoras de la Chureca), die bemerkenswerte Auswirkungen auf die politische und soziale Anerkennung der Gemeinschaft hatten. Kurz darauf wurde Nicaraguas nationale Müllsammler-Bewegung RedNica gegründet, um den geschätzten 13.500 informellen AbfallsammlerInnen des Landes eine einheitliche Stimme zu geben. Mittelfristig allerdings haben nur jene SammlerInnen profitiert, die in das formale System (Recyclinganlage) übernommen wurden, während sich für die anderen die Bedingungen verschlechtert haben. Sie sammeln in La Chureca weiterhin informell Abfall, was inzwischen als illegal gilt und höhere Risiken und mehr 
Repressionen mit sich bringt. Andere informelle SammlerInnen haben das Sammeln und Recyceln an andere Orte verlegt, was zu zahlreichen kleinen, unkontrollierten Deponien führt (EJAtlas 2020).

NICAMBIENTAL wurde 1995 gegründet und ist eine NGO, die sich für die Erhaltung und rationelle Nutzung von natürlichen Ressourcen, mit Schwerpunkt einheimische Waldarten einsetzt. Um einheimische und gefährdete Waldarten $\mathrm{zu}$ retten, wurde 2013 die Kampagne „Spenden Sie Ihre Elektroaltgeräte und retten Sie unser wertvolles Holz" gestartet, die von der Deutschen Gesellschaft für Zusammenarbeit (GIZ) gefördert wurde und sich auf die verantwortungsvolle Entsorgung von EAGs konzentriert. In den meisten Fällen arbeiten diese Gruppen von Menschen auf eigene Faust, ohne Sicherheitsmaßnahmen oder organisatorische Strukturen, die im Allgemeinen dazu beitragen, unter menschenwürdigen Bedingungen zu arbeiten. Sie haben keinen Zugang zu Schulungen und organisieren sich nicht in Verbänden oder Interessensgruppen. Viele von ihnen demontieren elektronische Geräte auf Straßen und in Parks ohne auf Gesundheits- und Sicherheitsrisiken zu achten; häufig lassen sie weniger wertvolle Teile oder Schrott auf öffentlichen Flächen oder an Flussufern zurück, wie z. B. Kathodenstrahlröhren von Fernsehern und Monitoren. In Nicaragua gibt es keine nennenswerte Industrie für die weitere Verarbeitung von Materialien aus der Demontage von EAGs.

\subsection{Ausbildung in Nicaragua}

In Nicaragua gibt es 4 staatliche und 51 private Universitäten. Die beiden Partneruniversitäten UCAN und ULSA sind Privatuniversitäten und befinden sich in Leon. Da es an den Universitäten keine Lehrveranstaltungen zu den Themen Umwelt und Abfallwirtschaft gibt, wurde im Zuge der Zusammenarbeit im LaWEEEda-Projekt (sh. Kap. 4) bei der Entwicklung der Lehrmaterialien auch Wert darauf gelegt, zusätzliches Basislehrmaterial im Umweltbereich zu entwickeln. Damit soll ein Grundverständnis geschaffen werden, auf dem tiefergehendes Wissen über Abfall- und insbesondere EAG-Management aufgebaut werden kann.

\section{Situation in Brasilien}

\subsection{Regelungen Abfallwirtschaft, Regelungen EAG}

Die Nationale Politik für Siedlungsabfälle (NPSW, eng. National Policy for Solid Waste) wurde durch das Bundesgesetz Nr. 12.305 (BRNS 2010) im August 2010 eingeführt und kann als Meilenstein für die Abfallwirtschaft in Brasilien angesehen werden. Die Ziele dieses Gesetzes sind die Vermeidung, Wiederverwendung, das Recycling, die Behandlung und die angemessene Entsorgung von Siedlungsabfällen, einschließlich Systemen zur Energierückgewinnung, um Schäden für die Umwelt und die öffentliche Gesundheit zu vermeiden. Dieses Gesetz verbietet die offene Deponierung von Siedlungsabfällen und es wurde festgelegt, dass alle Bundesstaaten und Städte ihre unkontrollierten Deponien bis 2014 geschlossen haben müssen. Nichtsdestotrotz hat sich die Situation in Bezug auf Siedlungsabfälle in Brasilien seit der Einführung des NPSW nur wenig verändert, und ein Großteil der Abfälle wird immer noch an ungeeignete Endziele geliefert (Gollo et al. 2014; Costa and Ferreira Dias 2020; Alfaia et al. 2017).

Als nächster Schritt wurde ein sogenanntes „Sektorales Abkommen“, eine Branchenregelung zur Festlegung der Verantwortlichkeiten in einem erweiterten Herstellerverantwortungssystem (EPR, eng. Extended Producer Responsibility) erarbeitet, allerdings wurde erst Ende 2019 ein Entwurf für dieses Sektorales Abkommen vorgelegt und diskutiert und der Prozess ist noch nicht abgeschlossen (Costa and Ferreira Dias 2020).

Das Dekret 7404/2010 (DPDR 2010), auf das sich das NPSW-Gesetz bezieht, definiert die Branchenvereinbarungen (BV) als das wichtigste Instrument für die Implementierung und den Betrieb von EAG-Rücknahmesystemen. Die BV sind Verträge, die zwischen Vertretern von Elektro(nik)geräte (EEE)-Herstellern, Importeuren, Distributoren und Einzelhändlern sowie der Regierung auf allen Ebenen zu unterzeichnen sind, für die Umsetzung der gemeinsamen Verantwortung für den Lebenszyklus von EEE. ABREE (Brasilianischer Verband für EEE Recycling) und FECOMERCIO (staatlicher Verband für den Handel mit Waren und Dienstleistungen) sind die derzeit größten Verbände, die durch die BV entstanden sind. Diese Ver- bände sind dazu berechtigt, ein oder mehrere EAG-Management-Unternehmen zu gründen, die institutionell für das Management und die Vergabe von Dienstleistungen verantwortlich sein werden und bereits für die Umsetzung der offiziellen EAG-Rücknahmesysteme verantwortlich sind (Souza et al. 2020).

Im Jahr 2013 hat der brasilianische Verband für technische Standards (ABNT) den Standard „Requirements for WEEE Reverse Manufacturing“ veröffentlicht, der als Referenz für den Umgang mit EAGs in brasilianischen Unternehmen gilt (ABNT 2013). Er legt Anforderungen für die Umsetzung, Aufrechterhaltung und kontinuierliche Verbesserung eines Managementsystems fest, das auf internationalen Standards wie der ISO-9000-Serie und ISO 14001 basiert. Einige der Eckpunkte des ABNT-Standards umfassen: Harmonisierung und standardisierte Dokumentation der statistischen Verwaltung in Bezug auf die Sammlung, Lagerung, Demontage und Vermarktung von Elektro- und Elektronik-Altgeräten; Management von gefährlichen Komponenten; die Nachvollziehbarkeit von Elektro- und Elektronik-Altgeräten vom Empfang bis zum endgültigen Bestimmungsort; verpflichtende Berichterstattung an KundInnen und Umweltbehörden (Souza et al. 2020).

\subsection{Abfallwirtschaft generell}

Brasilien ist das größte Land Lateinamerikas mit ca. 200 Mio. EinwohnerInnen (sechstgrößtes Land der Welt) und einer Fläche von $8.515 .767 \mathrm{~km}^{2}$ (fünftgrößtes Land der Welt). Die großen geografischen Entfernungen stellen neben dem Bevölkerungswachstum und der technologischen Entwicklung, die unvermeidlich zu einem erhöhten Abfallaufkommen führen, eine Herausforderung für das Abfallwirtschaftssystem in Brasilien dar.

Im Jahr 2017 fielen in Brasilien rund 78,4 Miot feste Siedlungsabfälle an, wobei der Anschlussgrad bei $91 \%$ lag. In den letzten 20 Jahren ist die Menge an Siedlungsabfällen um rund $31 \%$ gestiegen. Dies ist ein überproportionaler Anstieg im Vergleich zur Bevölkerungswachstumsrate $(7 \%)$ im gleichen Zeitraum (Alfaia et al. 2017). Rund 59\% der gesammelten Menge wurde in kontrollierten Deponien entsorgt, während die restlichen $41 \%$ in unkontrollierte Deponien („dumps“) mit niedrigen oder nichtexistierenden technischen Stan- 


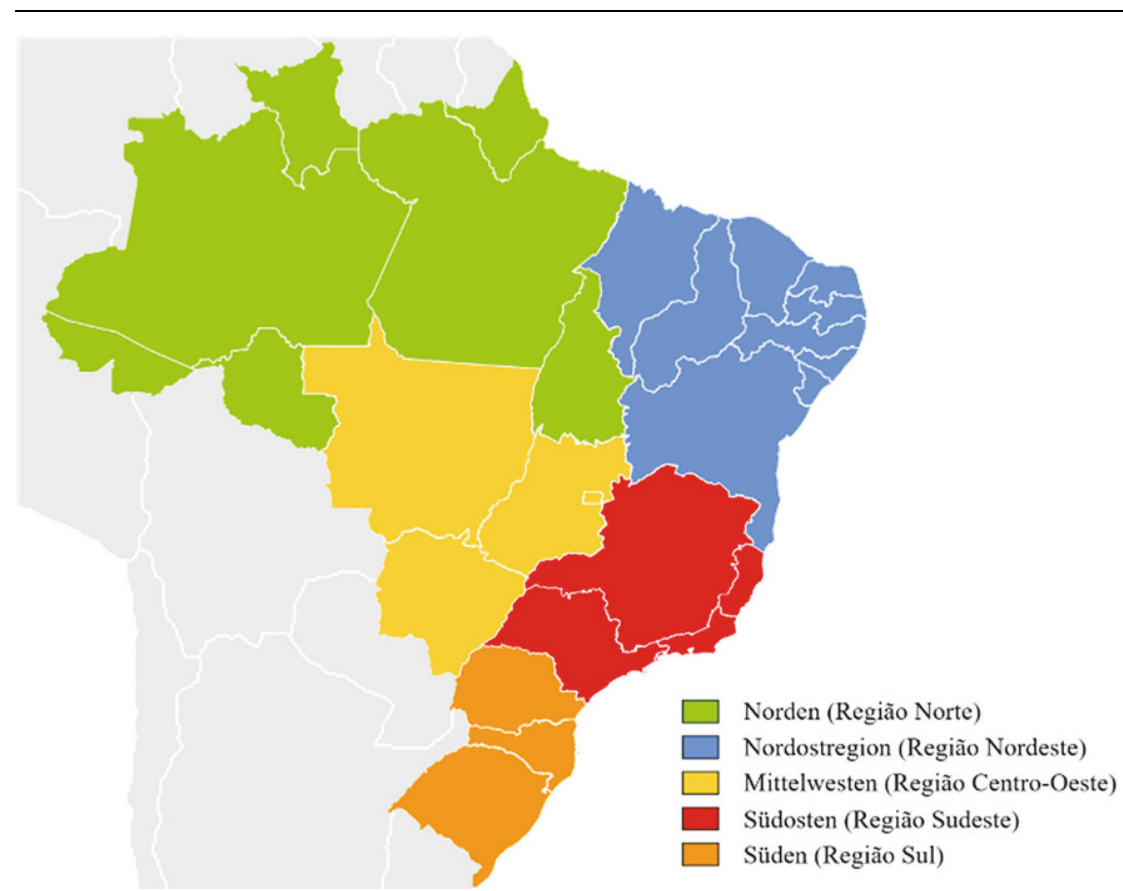

Abb. 2 Einteilung des brasilianischen Staatsgebiets in fünf geostatistische Regionen

dards geleitet wurden. Für ca. 7 Miot $(\sim 9 \%)$ der gesammelten Abfälle gibt es keinerlei Informationen über deren Entsorgung (Costa and Ferreira Dias 2020; Abb. 2).

In Bezug auf die Größe des Landes sind die starken Unterschiede im Entwicklungsniveau der Abfallwirtschaftssysteme zwischen den größten Ballungszentren Sao Paulo und Rio de Janeiro (Região Sudeste) und den bevölkerungsärmeren ländlichen Gebieten nicht überraschend. Laut Costa und Ferreira Dias (2020) sind rund $91 \%$ der Gemeinden derzeit mit einer Form des organisierten Sammelsystems abgedeckt, während rund $70 \%$ der Gemeinden entweder getrennte Sammlung oder zumindest eine Art der Wertstoffsammlung haben. Allerdings decken die Sammelsysteme oft nicht das gesamte Gemeindegebiet ab. Davon besonders betroffen sind sehr arme Stadtteile (die Favelas) und schwer erreichbare Gemeinden beispielsweise im Amazonasgebiet, v. a. Região Norte und Região Nordoste (Alfaia et al. 2017).

Die Wertstoffsammlung in Brasilien erfolgt auf zwei Arten: entweder durch die Bereitstellung von Abgabestellen, wo die Bevölkerung auf freiwilliger Basis ihre Wertstoffe abgeben kann, oder durch die informelle Sammlung durch Kooperativen (Alfaia et al. 2017). Eine Kooperative ist eine mehr oder weniger kohärente Vereinigung von
Siedlungsabfällen aussortiert und gesammelt werden.

\subsection{EAG-Aufkommen, Mengen}

Bisher basieren alle Daten in Bezug auf das Aufkommen, Sammlung und Behandlung von EAGs in Brasilien auf Schätzungsmethoden. Nach Forti et al. (2020) wird auf Basis der Kaufkraft der Bevölkerung geschätzt, dass 2019 in Brasilien 2,1 Mio t oder ca. 10,2 kg/EW.a an EAGs angefallen sind. Verglichen mit den 1,4 Miot aus dem Jahr 2014 ist das ein Anstieg von 9\% pro Jahr in den letzten fünf Jahren.

Basierend auf der verfügbaren Literatur und empirischen Erkenntnissen können die folgenden Massenströme von EAGs abgeleitet werden: Die wertvollen EAG-Fraktionen, die entweder durch Haus-zu-Haus-Sammlungen gesammelt oder von informellen SammlerInnen aus dem gemischten Siedlungsabfall aussortiert werden, werden an Kooperativen oder Schrotthändler verkauft. Die weniger wertvollen Fraktionen landen auf den unkontrollierten Deponien. Der alternative Weg in Gemeinden mit weiterentwickeltem EAG-Management umfasst die Sammlung durch das formale Sammelsystem (Souza et al. 2020; CEMPRE 2020; Araújo et al. 2012). Die gesammelten Geräte werden dann entweder mithilfe von Kooperativen oder durch das formale System aussortiert.

Die wertvollen Fraktionen werden erfasst und an Vorbehandler verkauft. Die Aluminium- und Eisenfraktionen werden meist im Land verwertet, während kupferhaltige Fraktionen entweder in Kupferhütten in Brasilien recycelt oder für den letzten Recyclingschritt nach Europa exportiert werden (Souza et al. 2020; CEMPRE 2020). Abhängig von den Marktpreisen der Rohmetalle nimmt auch der Umfang des informellen Recyclings von Metallen zu oder ab und umfasst einfache mechanische Recyclingtechniken für Eisenmetall, Kupfer und Aluminium oder hydrometallurgische Verfahren unter Verwendung von sauren Reagenzien aus der brasilianischen Bergbauindustrie.

\subsection{Der informelle Sektor und die Situation in Brasilien}

In einer globalen Perspektive spielt der informelle Sektor eine wesentliche Rolle in der Abfallwirtschaft, vor allem in Ländern mit einem nicht adäquat 
funktionierenden (formalen) Abfallwirtschaftssystem. Die Rückgewinnung von Materialien aus Abfällen stellt in Entwicklungs- und Schwellenländern einerseits eine Quelle für wertvolle Sekundärmaterialen dar, ist andererseits eine wesentliche Einnahmequelle für einen Teil der Bevölkerung und unterstützt die Agenden der formalen Abfallwirtschaft, die den steigenden Abfallmengen nicht gewachsen ist. Gründe für den Anstieg der Abfallmengen sind die rapide Verstädterung, die in unkontrolliertem und unorganisiertem Ausmaß stattfindet. Es fehlt an den nötigen Ressourcen die entsprechende Infrastruktur in sämtlichen Bereichen aufzubauen (Verkehr, Gesundheit u. Ä.), und resultiert nicht zuletzt in einer unzureichenden Sammlung und einer unsachgemäßen Entsorgung der anfallenden Abfälle (Medina 2000). Die formelle Abfallwirtschaft (öffentliche Hand oder private Unternehmen) ist nicht in der Lage, auf die steigenden Abfallmengen $\mathrm{zu}$ reagieren. Der informelle Sektor schließt diese Lücke und betreibt abfallwirtschaftliche Aktivitäten in unterschiedlichem Ausmaß. Meist sind sozial benachteiligte Bevölkerungsschichten in diesem Sektor tätig.

Der informelle Sektor in der Abfallwirtschaft wird nach Gunsilius et al. (2011) definiert als Einzelpersonen oder Unternehmen, die an Recyclingund Abfallwirtschaftsaktivitäten beteiligt sind, die aber nicht vom formellen Abfallwirtschaftssystem finanziert, anerkannt, unterstützt oder organisiert werden oder die gegen die Regelungen der offiziellen Stellen verstoßen oder in Konkurrenz zu diesen tätig sind. Eine weitere Charakteristik des informellen Sektors besteht darin, dass er ohne den Einsatz hoch entwickelter technischer Hilfsmittel auskommt und dadurch weniger störungsanfällig und kostengünstiger ist. Informelle Aktivitäten führen $\mathrm{zu}$ einer Ausweitung von abfallwirtschaftlichen Maßnahmen in ärmeren Gebieten. Dem gegenüber steht die „Informalität“ der Personen, was keinerlei arbeitsrechtliche oder soziale Absicherung (fixe Löhne, Kündigungsschutz, Gesundheitsvorsorge etc.) und häufig kritische Arbeitsbedingungen bedeutet. Informelle Aktivtäten finden entlang der gesamten Wertschöpfungskette statt; von der Sammlung bei Haushalten, bei Geschäften und Unternehmen, auf den Straßen und auf legalen und illegalen Deponien bis hin zu Aufbereitungsprozessen und schlussendlich dem Verkauf des Materials. Innerhalb des informellen Sektors gibt es unterschiedliche hierarchische Ebenen; spezialisierte Sammler bereiten die Abfälle auf, beispielsweise durch Waschen, Sortieren oder Demontage und verkaufen die Materialien an Zwischenhändler, die wiederum an einen übergeordneten Händler bzw. direkt an die Industrie die Sekundärrohstoffe verkaufen. Diese Zwischenhändler haben gute Verbindungen zu ihren Abnehmern in der Industrie und nehmen damit eine Monopolstellung ein, die sich in niedrigen Preisen für die Wertstoffe der informellen SammlerInnen niederschlägt.

Durch die Bildung von Kooperativen versuchen die informellen SammlerInnen, die Zwischenhändler zu umgehen und so den Mitgliedern höhere Preise zu bezahlen. Höhere Preise bedeuten wiederum ein höheres Einkommen und einen besseren Lebensstandard für die informellen SammlerInnen. Daneben gibt es weitere Vorteile; die Kooperativen verbessern das öffentliche Abfallwirtschaftssystem, ohne dass neue Personen angestellt und angelernt werden müssen und wertvolle Sekundärrohstoffe werden generiert. Teil einer Kooperative zu sein ändert auch den sozialen Status; man ist Teil einer Gruppe und die soziale Marginalisierung wird reduziert (Tirado-Soto und Zamberlan 2013). Andererseits ist die Gründung einer Kooperative relativ kostenintensiv (Tedde 2014) und bringt einen großen bürokratischen Aufwand mit sich. Fehlende finanzielle und logistische Unterstützung von der öffentlichen Hand erschwert die Gründung.

Es gibt zahlreiche Beispiele für erfolgreiche Kooperativen der informellen SammlerInnen. Eine der ersten Kooperativen in Lateinamerika entstand in Kolumbien, aber auch in Brasilien gibt es seit Jahren große Bemühungen, die Bildung solcher Kooperativen zu unterstützen. Der Zugang der Politik und der Behörden ist sehr unterschiedlich, Medina (2000) unterscheidet

- Verdrängung bzw. Unterdrückung: In vielen Ländern wird die Arbeit der informellen SammlerInnen nicht wertgeschätzt, sondern als Schande für die Stadt bzw. das Land verstanden. In der Folge wird das informelle Abfallsammeln zur illegalen Aktivität erklärt und mit Strafen belegt.
- Ignoranz: In einigen Ländern wird der informelle Sektor von den Behörden ignoriert. Die SammlerInnen werden weder bedrängt noch offiziell verfolgt, aber auch nicht unterstützt. Es wird keine Notwendigkeit gesehen, an der Situation etwas zu ändern.

- Geheime Absprachen: In einigen Fällen hat sich eine Art Einverständnis bzw. gegenseitige Abhängigkeit zwischen den Behörden und Teilen der informellen SammlerInnen (den „Bossen“) entwickelt. Als Beispiel wird von Medina (2000) Mexiko-Stadt genannt; dort haben sich über Jahrzehnte komplexe und zum Teil finanziell ertragreiche Beziehungen zwischen den Behörden, der Industrie, den Zwischenhändlern und informellen SammlerInnen entwickelt.

- Stimulierung: In einigen Ländern haben Regierungen begonnen, die ablehnende Haltung oder Gleichgültigkeit gegenüber den informellen SammlerInnen in Richtung Anerkennung der wirtschaftlichen, sozialen und ökologischen Vorteile zu ändern und in aktive Unterstützung umzuwandeln. Die unterstützenden Maßnahmen reichen von der Legalisierung der Aktivitäten, der Förderung der Bildung von Genossenschaften (wie in Indonesien), die Vergabe von Verträgen für die Sammlung von gemischten Abfällen und/oder Wertstoffen (in einigen kolumbianischen Städten), bis hin zur Bildung von öffentlich-privaten Partnerschaften zwischen lokalen Behörden und SammlerInnen (wie in einigen brasilianischen Städten).

Abfallwirtschaftspläne und abfallwirtschaftliche Entwicklungen haben oft massive Auswirkungen auf informelle SammlerInnen. Als Beispiel sei Bogotá (Kolumbien) genannt. Bis zum Bau war das Abfallsammeln auf Deponien üblich. Nach dem Bau einer kontrollierten Deponie wurde das informelle Sammeln dort verboten. Die SammlerInnen mussten auf die Straßen Bogotás ausweichen, um weiterhin ihren Lebensunterhalt $\mathrm{zu}$ verdienen. Die SammlerInnen mussten sich einen Karren zulegen um das Material transportieren zu können, sie mussten viel weitere Strecken zurücklegen, um eine für sie vernünftige Menge an Abfall zu sammeln, sie störten den Verkehr und wurden von Polizei und Straßengangs 
bedrängt. Die Produktivität (Menge an gesammelten Materialien pro Tag) war wesentlich geringer als auf der Deponie.

Nachfolgend werden einige Umsetzungen von Kooperativen in lateinamerikanischen Ländern vorgestellt. Wie Studien zeigen (Medina 2000; Schambe et al. 2018; Tirado-Soto und Zamberlan 2013), gibt es keine einheitliche Organisationsform von Kooperativen, sondern sehr verschiedene Ausgestaltungen in Bezug auf ihren rechtlichen Status, ihre Finanzierung und interne Organisation sowie ihre Funktionen, Aufgaben und sozialen Bestrebungen.

Eine der engagiertesten Initiativen in Bezug auf die Gründung von Kooperativen existiert in Kolumbien. Medina (2000) beschreibt, dass seit 1986 die Nichtregierungsorganisation „Fundacio'n Social“ informelle SammlerInnen bei der Gründung von Kooperativen unterstützt. Damals wurde eine offene Deponie in eine kontrollierte umgebaut und verdrängte 150 Familien, die bis dahin auf der Deponie Material gesammelt hatten. Die Stiftung half den vertriebenen AbfallsammlerInnen, eine Kooperative $\mathrm{zu}$ gründen. Als die positiven Auswirkungen dieser Bemühungen deutlich wurden, begann die Stiftung, auch in anderen Städten informelle SammlerInnen bei der Gründung von Kooperativen zu unterstützen. Im Jahr 1991 startete die Fundacio'n Social ihr nationales Recycling-Programm, das derzeit über 100 Kooperativen von AbfallsammlerInnen im ganzen Land umfasst. Die Kooperativen haben sich zu regionalen Vermarktungsverbänden zusammengeschlossen, was es ihnen ermöglicht, Wertstoffe in großen Mengen zu sammeln und zu verkaufen und dabei höhere Preise zu erzielen. Eine der erfolgreichsten Kooperativen ist Recuperar (2021) mit Sitz in Medellin. Sie wurde bereits 1983 gegründet, hat heute 1000 Mitglieder und ist im Reinigungsgewerbe sowie in Abfallsammlung und -recycling tätig. Die Mitglieder können von der Kooperative Kredite und Stipendien für Ausbildung erhalten, und haben eine Lebens- und Unfallversicherung.

Auch in Argentinien werden informelle SammlerInnen auf gesetzlicher Basis (Nationales Gesetz 25.916/04, lokale Gesetze für Buenos Aires 992/03 und 13.592/06) als relevante Akteure des Recyclingkreislaufs anerkannt. Im Allgemeinen nehmen diese Organisationen die Rechtsform einer Genossenschaft an und werden fortan als
„Recycler-Kooperativen“ bezeichnet, allerdings gibt es große Unterschiede in der Finanzierung (Subventionen und Erlöse aus Dienstleistungen) (Schambe et al. 2018).

Das informelle Sammeln von Abfällen wird auch in Mexiko von sozial benachteiligen Menschen ausgeführt, die damit ihren Lebensunterhalt verdienen. Es wird geschätzt, dass 800.000 Pepenadores in Mexiko arbeiten (Recuperar 2021). Eine der erfolgreichsten Kooperativen ist die Sociedad Cooperative de Seleccionadores de Materiales (SOCOSEMA). Mit der Gründung von SOCOSEMA 1975 wurden Zwischenhändler ausgebootet und das Einkommen der AbfallsammlerInnen stieg um fast das Zehnfache (Sánchez 2016). Eine weitere Initiative wurde von der Danone-Mexiko-Gruppe in Mexicali, Baja California initiiert und finanziert (Recuperar 2021). Das Ziel des Projektes „Pepenadores“ ist es, die informellen SammlerInnen von den Deponien wegzubringen und ihnen in einer Transfer- und Recyclingstation angemessene Arbeitsbedingungen $\mathrm{zu}$ bieten, damit sie wiederverwertbare Materialien, Schwerpunkt PET, trennen können. Es werden den ArbeiterInnen auch Unterkünfte bereitgestellt, soziale, medizinische und psychologische Betreuung angeboten sowie die Möglichkeit zur Schul- und Weiterbildung.

In Brasilien wurde in den letzten Jahren eine zunehmende Anzahl von Projekten und Initiativen umgesetzt, um die Arbeitsbedingungen der informellen SammlerInnen zu verbessern. Die ersten Kooperativen wurden in den 1990er-Jahren gegründet; inzwischen gibt es gemäß einer Umfrage des brasilianischen Instituts für Geografie und Statistik aus dem Jahr 2008684 Kooperativen von informellen AbfallsammlerInnen in Brasilien, davon allein 141 in Sao Paulo und 19 in Rio den Janeiro. Zusätzlich stellt das Gesetz 12.305 zur Nationalen Abfallpolitik (Política Nacional des Resíduos Solídoss) aus dem Jahr 2010 einen wesentlichen Schritt hin $\mathrm{zu}$ einer adäquaten Abfallsammlung und der Integration der informellen SammlerInnen in das Abfallwirtschaftssystem dar. Explizit wird in Art. 7 als eines der Ziele der nationalen Abfallpolitik die „Integration von Sammlern von wiederverwendbaren und wiederverwertbaren Materialien in Aktionen, die eine gemeinschaftliche Verantwortlichkeit für den Produktlebenszyklus entwickeln“ genannt. Zur Unterstüt- zung werden in Art. 8 „Anreize zur Bildung oder Entwicklung von Kooperativen oder anderen Formen des Verbandes von Sammlern von wiederverwendbaren oder wiederverwertbaren Materialien" als Instrumente gesehen. Die öffentliche Hand kann in Art. 42 Maßnahmen zur Finanzierung setzen, welche u. a. die „Einsetzung von Infrastruktur und Ankauf von Geräten für Kooperativen oder andere Formen von Sammlern (...) bestehend aus Personen mit niedrigem Einkommen“ fördern.

Im LaWEEEda-Projekt wurden Kooperativen für die Entwicklung und Durchführung von CPD-Kursen (Ausbildungskurse zur beruflichen Weiterbildung) einbezogen. In Rio de Janeiro wurden mit COOPAMA und ACAMJG zwei etablierte und engagierte Partner gefunden, in Sao Paulo wurde Coopermiti als strategischer Partner gewonnen.

Ein Beispiel für eine Kooperative in der Verwertung von Elektroaltgeräten ist COOPAMA in Rio de Janeiro. Die MitarbeiterInnen dieser Einrichtung (gegründet 2004) haben eine Ausbildung für die Demontage und die Verwertung von Elektroaltgeräten und wurden von der Stadt als Verwerter anerkannt. COOPAMA hat ein Team von 11 ausgebildeten MitarbeiterInnen im Bereich Demontage und bearbeitet $240 \mathrm{t}$ Elektroaltgeräte und andere Abfälle pro Monat, die aus einem Netzwerk von Partnern im Bereich Elektrohandel stammen. Ein anderes Beispiel ist die Kooperative Coopertech in in Sao Jose dos Campos, die ebenfalls mit der Kommune zusammenarbeitet und Elektroaltgeräte aus der städtischen Sammlung sowie von großen Unternehmen übernimmt, durchschnittlich $5 t /$ Monat. Diese Kooperative war unter den ersten Unternehmen, die Brasilianische Standards für EAG umgesetzt haben.

ACAMJG - Associação dos Catadores do Aterro Metropolitano de Jardim Gramacho - ist eine Kooperative, die auf der Deponie Jardim Gramacho, Rio de Janeiro entstanden ist. Diese Deponie, die eine der größten der Welt war, wurde 2012 nach 34 Jahren geschlossen, es leben aber nach wie vor viele AbfallsammlerInnen auf und von der Deponie. Die Mitglieder werden im Austausch für die Wertstoffe, die sie sammeln und sortieren, bezahlt. Vor einigen Jahren hat ACAMJG durch den Dokumentarfilm „Waste Land“ (von Vik Muniz) Bekanntheit erlangt. Der Leiter 
der Kooperative, Sebastião Santos, hat mit 25 Jahren die ACAMJG gegründet, nachdem er selbst jahrelang als Abfallsammler auf der Deponie seinen Lebensunterhalt bestreiten musste. Er ist ein sehr engagierter Vertreter der SammlerInnen und aktiv um die Bekanntmachung und Verbesserung der Situation der SammlerInnen bemüht. Laut Santos sind nur $4 \%$ der informellen SammlerInnen in Kooperativen oder Verbänden organisiert; $96 \%$ sind somit sozial und wirtschaftlich noch weiter ausgeschlossen, trotz des Gesetzes 12.305 aus dem Jahr 2010, das die Einbindung der informellen SammlerInnen vorsieht.

Zusammenfassend kann man über Kooperativen sagen, dass sich darin - außer durch die einheitliche Bezeichnung - kaum die gleichen Akteure, Interessen oder Praktiken, die vielfältig und in einigen Fällen widersprüchlich sind, unter demselben Label wiederfinden. Meist ist zutreffend, dass in den Kooperativen hauptsächlich Menschen aus verarmten Schichten tätig sind, die durch das Sammeln von Abfällen in einer Kooperative eine Möglichkeit gefunden haben, Einkommen und Lebensbedingungen für sich und ihre Familien sowie ihren sozialen Status etwas zu verbessern.

\subsection{Bestehende Ausbildungen in Brasilien}

Anders als in Nicaragua gibt es in Brasilien zahlreiche universitäre und berufsbegleitende Ausbildungsmöglichkeiten im Bereich Umwelttechnik. Beispielsweise gibt es in Sao Paulo 14 Universitäten, an denen eine Ausbildung im Umweltbereich angeboten wird, an drei Einrichtungen gibt es CPD-Kurse. In Rio de Janeiro Staat gibt es insgesamt 9 Universitäten; Ausbildungen im Umweltbereich finden vorwiegend im Bereich Umwelttechnik, Umweltmanagement und Abwassertechnik statt.

\section{Ausbildungsprogramme}

Ein Beispiel für ein Ausbildungsprogramm im Bereich EAG stellen die im LaWEEEda-Projekt entwickelten Kurse dar. Im Zuge dieses Erasmus+Projekts wurde ein ausbildungsorientiertes Netzwerk von Europäischen und Lateinamerikanischen Universitäten geschaffen. In Brasilien sind das Universidade Estadual Paulista „Julio de Mesquita Filho“ (UNESP) in Sao Jose dos Campos und die Universidad Federal de Rio de Janeiro (UFRJ). Die Partneruniversitäten in Nicaragua sind zwei Universitäten in Leon, die Universidad Tecnológica La Salle (ULSA) und die Universidad Cristiana Autónoma de Nicaragua (UCAN). Von Europäischer Seite sind die TU Hamburg, die University of Northampton, UK und die Universität für Bodenkultur Wien Partner, letztere als Koordinator des Projekts. Weiters haben vier Wirtschaftspartner mit praktischem Wissen und Marktkenntnis dazu beigetragen, ein maßgeschneidertes Ausbildungsprogramm zu etablieren. Am Weg dorthin wurde zunächst die Ausgangslage in beiden Ländern analysiert. Dazu wurden abfallwirtschaftliche Rahmenbedingungen, der bestehende Rechtsrahmen sowie existierende Ausbildungsschienen erhoben. Im Zuge eines Stakeholderprozesses wurde der Bedarf an Ausbildung, insbesondere in der berufsbegleitenden Ausbildung detailliert erhoben. Darauf basierend wurden für die Ausbildung in Nicaragua neben dem Umgang mit EAG auch abfallwirtschaftliche Grundlagen einbezogen, während für die Kurse in Brasilien der Fokus stärker auf technische und organisatorische Maßnahmen im Umgang mit EAG gelegt wurde. Neben technischen Aspekten werden im Trainingsprogramm auch Unternehmensführung und Arbeitssicherheit vermittelt. Die beiden Zielgruppen für das Trainingsprogramm sind ExpertInnen und PraktikerInnen aus dem Bereich Industrie, Handel und öffentliche Einrichtungen, die im Sinne der beruflichen Weiterbildung spezifisches Wissen im Umgang mit diesem Abfallstrom benötigen. Daneben wird das Thema Elektroaltgeräte auch in das Ausbildungsprofil der beteiligten Universitäten aufgenommen, in den meisten Fällen in Ingenieurausbildungen, wobei hier mehr Theorie, aber auch praxisbezogenes Wissen vermittelt wird.

Für die universitäre Ausbildung wurden drei Module entwickelt. Modul 1 Abfallwirtschaft und Umweltauswirkungen behandelt die Auswirkungen des nicht sachgemäßen Umgangs mit Abfällen auf Gesundheit und Umwelt. Themen sind kommunale Abfallmengen und Zusammensetzung, Sammelsysteme für kommunale Abfälle, die Verwertung von Altstoffen, der Umgang mit gefährlichen Abfällen, die Technologie der Behandlung von Abfällen sowie finanzielle Aspekte (Kosten und
Gebühren) der kommunalen Abfallwirtschaft. Modul 2 - Elektroaltgeräte Hintergrundwissen umfasst die Gesetzgebung für EAG, Entstehung und Aufkommen von EAG, Wiederverwertung und Reparatur, Sammlung, Materialzusammensetzung, Technologie der Aufbereitung wie mechanische Aufbereitung, die selektive Behandlung bestimmter Bauteile sowie die Behandlung nicht verwertbarer Bauteile und Materialien. Modul 3 - Elektroaltgeräte Praxiswissen umfasst die Kostenkalkulation der Verwertung von Elektroaltgeräten, die Entwicklung von Businessplänen, die Anlagenplanung, die Analytik von Bauteilen und Materialien im Labor und vor Ort, die Demontagepraxis sowie die Aktivitäten des informellen Recyclingsektors.

Das wichtigste Ergebnis sind vier Trainingszentren für Elektroaltgeräte an den Partneruniversitäten in Brasilien und Nicaragua. Diese Trainingszentren widmen sich der praktischen und theoretischen Vermittlung von Kenntnissen entlang der Recyclingkette von Elektroaltgeräten. Als Ergebnis des Projekts wurden umfangreiche Trainingsmaterialien öffentlich verfügbar gemacht und zwar auf Portugiesisch, Spanisch und auch Englisch. Weiters wurden in Zusammenarbeit mit dem Demontage- und Recyclingzentrum Wien (DRZ) Videos produziert, in denen die Demontage und Schadstoffentfrachtung von Elektroaltgeräten erläutert wird. Diese Videos sind ebenfalls in den drei Sprachen verfügbar. Im September 2019 wurden die Trainingszentren offiziell eröffnet und haben ihren Betrieb aufgenommen. Details zum Projekt, zukünftige Trainings und Veranstaltungen sowie Materialien und Videos sind über die Projektseite zugänglich. Details sh. http://laweeedaeprints.iolcus.co.uk/.

\section{Ausblick}

In Anbetracht der steigenden Mengen ist die fachgerechte Behandlung von EAG ein relevantes Thema für Lateinamerika. Der Entwicklungsstand - wie die beiden Länderbeispiele Nicaragua und Brasilien zeigen - ist sehr heterogen. Da formelle Strukturen zur Sammlung und Behandlung fehlen, sollte bei der Suche nach adäquaten Lösungen der informelle Sektor mitbedacht werden, der in beiden Ländern eine zentrale Rolle spielt.

Ausbildung spielt bei der Entwicklung und Umsetzung von Strategien für 
das EAG-Recycling eine große Rolle. Am Beispiel eines Kooperationsprojekts mit Universitäten und Nicaragua und Brasilien wird gezeigt, wie die akademische Ausbildung und die berufsbegleitende Ausbildung im Bereich Abfallwirtschaft und Recycling von Elektroaltgeräten zu dieser Entwicklung beitragen kann.

Danksagung Das zugrunde liegende Projekt („LaWEEEda - Latin AmericanEuropean network for research, development and analysis of waste from electrical and eletronic equipment") wurde von Dr. Stefan Salhofer geleitet und von der EACEA im Programm ERASMUS+ (Projektnummer 573517) finanziert. Besonderer Dank geht an die Partner in diesem Kooperationsprojekt.
Funding Open access funding provided by University of Natural Resources and Life Sciences Vienna (BOKU).

Open Access Dieser Artikel wird unter der Creative Commons Namensnennung 4.0 International Lizenz veröffentlicht, welche die Nutzung, Vervielfältigung, Bearbeitung, Verbreitung und Wiedergabe in jeglichem Medium und Format erlaubt, sofern Sie den/die ursprünglichen Autor(en) und die Quelle ordnungsgemäß nennen, einen Link zur Creative Commons Lizenz beifügen und angeben, ob Änderungen vorgenommen wurden.
Die in diesem Artikel enthaltenen Bilder und sonstiges Drittmaterial unterliegen ebenfalls der genannten Creative Commons Lizenz, sofern sich aus der Abbildungslegende nichts anderes ergibt. Sofern das betreffende Material nicht unter der genannten Creative Commons Lizenz steht und die betreffende Handlung nicht nach gesetzlichen Vorschriften erlaubt ist, ist für die oben aufgeführten Weiterverwendungen des Materials die Einwilligung des jeweiligen Rechteinhabers einzuholen.

Weitere Details zur Lizenz entnehmen Sie bitte der Lizenzinformation auf http://creativecommons.org/licenses/ by/4.0/deed.de.
ABIPET (2016): Censo da reciclagem de PET no Brasil. Associação Brasileira da Indústria do PET. Available at: http://www.abipet.org.br/index. $\mathrm{html}$ ? ethod=mostrardownloads\&categoria.id $=3$. Last accessed March 2021

ABNT (2013): ABNT NBR 16156 - Waste electrical and electronic equipment: requirements for the activity of reverse manufacturing.

Alfaia, R. G. d. S. M., Costa, A. M., \& Campos, J. C. (2017): Municipal solid waste in Brazil: A review. (1096-3669 (Electronic)).

ANAP (2016): Evolution of paper recovery rate in Brazil. Available at: http://anap.org.br/anap/ wp-content/uploads/2016/09/ANAP-Relat\%C3 \%B3rio-Anual-2015\%E2\%80\%932016.pdf. Last accessed March 2021

ANdD (2014): Ley Especial de Gestión Integral de Residuos y Desechos Sólidos Peligrosos y no Peligrosos. A. N.d. Nicaragua. Available at: http://legislacion.asamblea.gob.ni/SILEG/ Iniciativas.nsf/0/e6b32d91d9b2f6e8062578a3007 $1 \mathrm{fddd} /$ \$FILE/Articulado\%20en\%20Dictamen \%20 Ley\%20General\%20de\%20Gestion\%20Res\%20 Solidos.pdf. Last accessed March 2021 Araújo, M. G., Magrini, A., Mahler, C. F., \& Bilitewski, B. (2012): A model for estimation of potential generation of waste electrical and electronic equipment in Brazil. Waste Management, 32(2), 335-342. https://doi.org/10.1016/j. wasman.2011.09.020.

Arce, E. M., Diego, D., Espinoza, P. T., Faure, M. S., \& Terraza, H. (2010): Regional Evaluation on Urban Solid Waste Management in Latin America and the Caribbean: 2010 Report. Available at: https://publications.iadb.org/en/regionalevaluation-urban-solid-waste-managementlatin-america-and-caribbean-2010-report. Last accessed March 2021

Baldé, C. P., Wang, F., Kuehr, R., \& Huisman,

J. (2015): The global e-waste monitor-2014. 2016/04/01/. United Nations University, IAS - SCYC Bonn, Germany. Available at: https://i.unu.edu/ media/unu.edu/news/52624/UNU-1stGlobalE-Waste-Monitor-2014-small.pdf.

Last accessed March 2021

BRNS (2010): Law No. 12.305 on the National Policy on Solid Waste Management.. National Congress. Available at: http://www.braziliannr.com/ brazilian-environmental-legislation/law-no12305-brazilian-national-policy-solid-waste/. Last accessed April 2021
CEMPRE (2020): Ciclosoft 2020 - Die größte Studie zur selektiven Sammlung und Rücknahmelogistik in Brasilien. https://cempre.org.br/ pesquisa-ciclosoft/. Last accessed March 2021 Costa, I. M., \& Ferreira Dias, M. (2020): Evolution on the solid urban waste management in Brazil: A portrait of the Northeast Region. Energy Reports, 6, 878-884. https://doi.org/10.1016/j.egyr 2019.11.033

DPDR (2010): DECRETO No 7.404 - que institui a Política Nacional de Resíduos Sólidos, cria o Comité Interministerial para a Política Nacional de Resíduos Sólidos e o Comité de Orientação para a Implementação de Sistemas Logísticos Invertidos, e dita outras disposições. P. d. República. Available at: http://www.planalto.gov.br/ ccivil_03/_ato2007-2010/2010/decreto/d7404. htm. Last accessed March 2021

EJAtlas (2020): La Chureca dumpsite enclosure, Managua, Nicaragua. EJAtlas-Global Atlas of Environmental Justice. https://www.ejatlas. org/conflict/displacement- of-waste-pickersthrough-la-chureca-dumpsite-enclosuremanagua-nicaragua. Last accessed April 2021 Forti, V., Balde, C. P., Kuehr, R., \& Bel, G. (2020): The Global E-waste Monitor 2020: Quantities, flows and the circular economy potential. Bonn, Geneva and Rotterdam: United Nations University/United Nations Institute for Training and Research and International Telecommunication Union.

Gollo, R., Braconi, M., Rossin, C., Nishi, J., \& Michellis, C. (2014): Três anos após a regulamentação da Política Nacional de Resíduos Sólidos (PNRS): seus gargalos e superações. PwC. Available at: http://ablp.org.br/pdf/Estudo_Selur 2014_final.pdf. Last accessed March 2021 Gunsilius, E., Chaturvedi, B., Scheinberg, A., Coad, A., \& Cortes, S. G. (2011): The Economics of the Informal Sector in Solid Waste , Management. CWG-Collaborative Working Group on Solid Waste Management in Low- and Middle-income Countries and GIZ - Deutsche Gesellschaft für Internationale Zusammenarbeit (GIZ) GmbH. Available at: https://www. giz.de/de/downloads/giz2011-cwg-bookleteconomicaspects.pdf. Last accessed March 2021 IADB (2011): Regional evaluation on urban solid waste management in Latin America and the Caribbean-2010 Report. Inter-American Development Bank. Available at: https://publications. iadb.org/en/regional-evaluation-urban-solid- waste-management-latin-america-andcaribbean-2010-report. Last accessed March 2021

Kaza, S., Yao, L. C., Bhada-Tata, P., \& Van Woerden, F. (2018): What a Waste 2.0: A Global Snapshot of Solid Waste Management to 2050. Urban Development;. Washington, DC: World Bank. (C) World Bank. DC: World Bank: World Bank.

laWEEEda (2017): Report on social, gender, ethical, regional and national aspects.

MAVDT (2010): RESOLUCIÓN 1512 DE $2010-$ Por la cual se establecen los Sistemas de Recolección Selectiva y Gestión Ambiental de Residuos de Computadores y/o Periféricos y se adoptan otras disposiciones. V. y. D. T. El Ministro de Ambiente. Available at: http://www.bogotaturismo gov.co/sites/intranet.bogotaturismo.gov.co/ files/RESOLUCI\%C3\%93N\%201512\%20DE\% 202010.pdf. Last accessed March 2021 Medina, M. (2000): Scavenger cooperatives in Asia and Latin America. Resources, Conservation and Recycling, 31(1), 51-69. https://doi.org/ 10.1016/S0921-3449(00)00071-9.

Recuperar (2021): QUIÉNES SOMOS Reseña histórica. http://recuperar.com.co/quienessomos.html. Last accessed March 2021

Schambe, P. J., Tagliafico, J. P., \& Sarandón, F (2018): Recyclers' cooperatives in Latin America. Cooperativas de las Americas. Available at: https://www.aciamericas.coop/IMG/pdf/1. 1_p._schamber_f._sarandon_en_con_firma_en. pdf. Last accessed March 2021

SGIRS (2005): Decretos Ejecutivos - La Política Nacional sobre la Gestión Integral de Residuos Sólidos. E. P. d. l. R. d. Nicaragua 47-2005 Available at: http://legislacion.asamblea.gob.ni/ normaweb.nsf/b34f77cd9d23625e06257265005 $\mathrm{d} 21 \mathrm{fa} / \mathrm{d} 132318726051846062570 \mathrm{ab} 0064017 \mathrm{~d}$ ? OpenDocument. Last accessed March 2021

Souza, R. G., Prasad, M. N. V., Vithanage, M., \& Borthakur, A. (2020): 16-E-waste situation and current practices in Brazil. Handbook of Electronic Waste Management (pp. 377-396). Butterworth-Heinemann. https://doi.org/10. 1016/B978-0-12-817030-4.00009-7.

Sánchez, T. E. (2016): Mexico City recognizes the rights of informal waste pickers. https:// globalrec.org/2016/08/22/mexico-city-recog nizes-the-rights-of-informal-waste-pickers/.

Last accessed March 2021 
Tedde, A. (2014): Solid waste management and the strategic role of waste-pickers: scavengers' cooperatives in Rio de Janeiro. Leiden University Tirado-Soto, M. M., \& Zamberlan, F. L. (2013): Networks of recyclable material waste-picker's cooperatives: An alternative for the solid waste management in the city of Rio de Janeiro. Waste Management, 33(4), 1004-1012. https://doi.org/ 10.1016/j.wasman.2012.09.025.

Hinweis des Verlags Der Verlag bleibt in Hinblick auf geografische Zuordnungen und Gebietsbezeichnungen in veröffentlichten Karten und Institutsadressen neutral. 